\title{
ON THE LIMITING BEHAVIOR OF A HARMONIC OSCILLATOR WITH RANDOM EXTERNAL DISTURBANCE
}

\author{
G.L. KULINICH \\ Kiev University \\ Department of Mathematics \\ 64 Volodymyrska St. \\ Kiev 252017, Ukraine
}

(Received October, 1994; Revised March, 1995)

\begin{abstract}
This paper deals with the limiting behavior of a harmonic oscillator under the external random disturbance that is a process of the white noise type. Influence of noises is investigated in resonance and non-resonance cases.
\end{abstract}

Key words: Harmonic Oscillator, Instantaneous Energy, Differential Equation of the Second Order, Itô Stochastic Differential Equation.

AMS (MOS) subject classifications: $60 \mathrm{H} 10$.

\section{Introduction}

We investigate the harmonic oscillator as a system of motion described by a linear differential equation of the second order

$$
m \ddot{u}(t)+k u(t)=q(t) \text { while } m>0 \text { and } k>0,
$$

where $q(t)$ is an external disturbance force. In the case, where $q(t)$ is a nonrandom periodic function, the instantaneous energy of the oscillator $\varepsilon(t)=\frac{1}{2}\left[k u^{2}(t)+m \dot{u}^{2}(t)\right]$ is bounded if the period of the function $q(t)$ is not equal to $2 \pi \sqrt{m / k}$ and $\varepsilon(t) \sim t^{2}$ as $t \rightarrow \infty$ if period of function $q(t)$ is equal to $2 \pi \sqrt{m / k}$ (resonance).

A model of the random harmonic oscillator with $\varepsilon(t) \sim t$ as $t \rightarrow \infty$ was considered by Papanicolau [8] for the case when $q(t)$ is a stationary random process; a model in which $\ln \varepsilon(t) \sim t \rightarrow \infty$ was considered by Bendersky and Pastur [1] for the case when $q(t)=0$ and $k=k(t)$ is a stationary random process; a model in which $\varepsilon(t) \sim \sqrt{t}$ as $t \rightarrow \infty$ was considered by Kulinich [7] for the case when $q(t)=g(w(t)) \dot{w}(t)$, with $\dot{w}(t)$ as a "white" noise, $g(x)$ a nonrandom function and $g^{2}(x)$ integrable over $\mathbb{R}$.

In the present paper, we consider the external random disturbance of the type $q(t)$ $=f(t) g(w(t)) \dot{w}(t)$, where $f(t)$ and $g(x)$ are nonrandom functions and $f^{2}(t)$ is a periodic function with the period $2 L$.

The limiting behavior (for $t \rightarrow \infty$ ) of the joint distribution of the random variables $(u(t), \dot{u}(t)$ ) the distribution of the random variable $\varepsilon(t)$ is investigated in the following cases:

1) $2 L \neq 2 \pi \sqrt{m / k}$; 2) $2 L=2 \pi \sqrt{m / k}$.

It is shown in particular that $\varepsilon(t) \sim t$ if $g^{2}(x) \sim b \neq 0$ as $|x| \rightarrow \infty$ (Theorem 1) and 
$E \varepsilon(t) \sim t^{\frac{\alpha+1}{2}}$ if $g^{2}(x) \sim b(x)|x|^{\alpha-1}$, while $\alpha>0$ and $b(x)=b_{1}$ for $x>0$, and $b(x)=b_{2}$ for $x<0$ (corollary of Theorem 2).

Let $u(t)$ be the distance of a particle from its equilibrium position. We assume that the particle has mass $m$ and that it is fastened to an immobile support by a spring with the coefficient of stiffness $k$. Then $u(t)$ satisfies the following equation:

$$
m \ddot{u}(t)+k u(t)=q(t) \text { while } u(0)=u_{0} \text { and } \dot{u}(0)=\dot{u}_{0}\left(\dot{u} \equiv \frac{d}{d t} u\right) .
$$

Here $q(t)$ is an external force, $u_{0}$ is an initial position and $\dot{u}_{0}$ is an initial velocity of the particle. We assume, then, that $u_{0}=0, \dot{u}_{0}=0$ and $q(t)=f(t) g(w(t)) \dot{w}(t)$, where $w(t)$ is a Wiener process. In this case, equation (1) can be considered as a system of stochastic Itô equations:

$$
\left\{\begin{array}{c}
m d \dot{u}(t)=-k u(t) d t+f(t) g(w(t)) d w(t) \\
d u(t)=\dot{u}(t) d t
\end{array}\right.
$$

Lemma: Let function $f(t)$ satisfy the condition, $\left|\int_{0}^{t} f(s) d s\right| \leq C$, for every finite $t$, and let $g(x)$ have the second derivative $g^{\prime \prime}(x)$ almost everywhere while $\int_{0}^{x}\left|g^{\prime \prime}(v)\right| d v=o\left(|x|^{\alpha}\right)$ as
$|x| \rightarrow \infty$ with $\alpha>0$. Then,

$$
\lim _{t \rightarrow \infty} t^{-\frac{\alpha+1}{2}} E\left|\int_{0}^{t} f(s) g(w(s)) d s\right|=0,
$$

where $w(t)$ is a Wiener process.

Proof: Since the functions $f(t)$ and $g^{\prime \prime}(x)$ are integrable over every bounded domain, because of Krylov [5], we can apply Itô's formula to the process $\Phi(t, w(t))$, where $\Phi(t, x)=\int_{0}^{t} f(s) d s g(x)$,
and obtain

$$
\begin{gathered}
\int_{0}^{t} f(s) g(w(s)) d s=\int_{0}^{t} f(s) d s g(w(t))-\int_{0}^{t}\left[\int_{0}^{s} f\left(s_{1}\right) d s_{1}\right] g^{\prime}(w(s)) d w(s) \\
-\frac{1}{2} \int_{0}^{t}\left[\int_{0}^{s} f\left(s_{1}\right) d s_{1}\right] g^{\prime \prime}(w(s)) d s=I_{1}(t)+I_{2}(t)+I_{3}(t) .
\end{gathered}
$$

It is easy to see that the following inequalities hold true:

$$
\begin{gathered}
t^{-\frac{\alpha+1}{2}} E\left|I_{1}(t)\right| \leq C t^{-\frac{\alpha+1}{2}} E|g(w(t))| \\
E\left(t^{-\frac{\alpha+1}{2}} I_{2}(t)\right)^{2}=t^{-(\alpha+1)} E \int_{0}^{t}\left[\int_{0}^{s} f\left(s_{1}\right) d s_{1} g^{\prime}(w(s))\right]^{2} d s \\
\leq C^{2} t^{-(\alpha+1)} E \int_{0}^{t}\left[g^{\prime}(w(s))\right]^{2} d s \\
t^{-\frac{\alpha+1}{2}} E\left|I_{3}(t)\right| \leq \frac{1}{2} C t^{-\frac{\alpha+1}{2}} E \int_{0}^{t}\left|g^{\prime \prime}(w(s))\right| d s .
\end{gathered}
$$


Next, applying the Itô formula to the processes $\Phi(w(t))$ and $\Phi_{1}(w(t))$, where

$$
\Phi(x)=2 \int_{0}^{x}\left[\int_{0}^{z}\left(g^{\prime}(v)\right)^{2} d v\right] d z \text { and } \Phi_{1}(x)=2 \int_{0}^{x}\left[\int_{0}^{z}\left|g^{\prime \prime}(v)\right| d v\right] d z,
$$

we obtain the equations

and

$$
t^{-(\alpha+1)} E \int_{0}^{t}\left[g^{\prime}(w(s))\right]^{2} d s=t^{-(\alpha+1)} E \Phi(w(t))
$$

$$
t^{-\frac{\alpha+1}{2}} E \int_{0}^{t}\left|g^{\prime \prime}(w(s))\right| d s=t^{-\frac{\alpha+1}{2}} E \Phi_{1}(w(t)) .
$$

The conditions of the Lemma require that $g(x)=o\left(|x|^{\alpha+1}\right), \Phi(x)=o\left(|x|^{2 \alpha+1}\right)$ and $\Phi_{1}(x)=$ $o\left(|x|^{\alpha+1}\right)$. When we take into account that $w(t) t^{-\frac{1}{2}}$ for every $t>0$ is standard normal it is easy to ensure that $E \frac{|g(w(t))|}{\frac{\alpha+1}{2}} \rightarrow 0, E \frac{\Phi(w(t)}{t^{(\alpha+1)}} \rightarrow 0$ and $E \frac{\Phi_{1}(w(t))}{\frac{\alpha+1}{2}} \rightarrow 0$ as $t \rightarrow \infty$. These convergences along with (3) and (4) yield the Lemma.

In what follows, we assume that $f(t)$ in the equations is a continuously differentiable function and that $f^{2}(t)$ has period $2 L$. Let us denote

$$
\begin{gathered}
a_{0}=\frac{1}{4 L} \int_{0}^{2 L} f^{2}(t) d t, \quad c_{0}=\frac{1}{4 L} \int_{0}^{2 L} f^{2}(t) \cos (2 \sqrt{k / m} t) d t \\
a_{1}=a_{0}+c_{0}, \quad a_{2}=a_{0}-c_{0} \quad \text { and } \quad a_{3}=\frac{1}{4 L} \int_{0}^{2 L} f^{2}(t) \sin (2 \sqrt{k / m} t) d t .
\end{gathered}
$$

Theorem 1: Let the function $g(x)$ in equation (2) have a second derivative with

$$
\lim _{|x| \rightarrow \infty} \frac{1}{x} \int_{0}^{x} g^{2}(v) d v=b \quad \text { and } \quad \lim _{|x| \rightarrow \infty} \frac{1}{x} \int_{0}^{x}\left|g^{\prime}(v)+g(v) g^{\prime \prime}(v)\right| d v=0 .
$$

1. Suppose $2 L \neq n \pi \sqrt{m / k}$ for any $n=1,2, \ldots$ or $2 L=n_{0} \pi \sqrt{m / k}$ and at the same time, $c_{0}=0$ and $a_{3}=0$. Then the following hold:

a) The joint distribution of the random variables $(u(t) / \sqrt{t}, \dot{u}(t) / \sqrt{t})$, as $t \rightarrow \infty$, converges to the distribution of $\left(\sqrt{\frac{a_{0} b}{k m}} \zeta_{1}, \frac{\sqrt{a_{0} b}}{m} \zeta_{2}\right)$, where $\zeta_{1}$ and $\zeta_{2}$ are independent standard normal random variables.

b) The distribution of the random variable $t^{-1} \varepsilon(t)$, as $t \rightarrow \infty$, converges to the exponential distribution with the parameter $m\left(a_{0} b\right)^{-1}$.

2. Suppose $2 L=n_{0} \pi \sqrt{m / k}$ and that $c_{0} \neq 0$ or $a_{3} \neq 0$. Then the following hold:

a) $P\left\{\frac{u(t)}{\sqrt{t}}<x_{1}, \frac{\dot{u}(t)}{\sqrt{t}}<x_{2}\right\}-F_{t}\left(x_{1}, x_{2}\right) \rightarrow 0$, where for each $t>0, F_{t}\left(x_{1}, x_{2}\right)$ is bivariate normal with the density:

$$
\rho_{t}\left(x_{1}, x_{2}\right)=\frac{1}{2 \pi \sigma_{1} \sigma_{2} \sqrt{1-r^{2}}} \exp \left\{-\frac{1}{2\left(1-r^{2}\right)}\left[A x_{1}^{2}-2 B x_{1} x_{2}+C x_{2}^{2}\right]\right\}
$$


where

$$
\begin{gathered}
A=\frac{\sin ^{2} \alpha}{\sigma_{1}^{2}}+2 r \frac{\sin \alpha \cos \alpha}{\sigma_{1} \sigma_{2}}+\frac{\cos ^{2} \alpha}{\sigma_{2}^{2}}, \quad B=-\frac{\sin \alpha \cos \alpha}{\sigma_{1}^{2}}+r \frac{\sin ^{2} \alpha-\cos ^{2} \alpha}{\sigma_{1} \sigma_{2}}+\frac{\sin \alpha \cos \alpha}{\sigma_{2}^{2}}, \\
C=\frac{\cos ^{2} \alpha}{\sigma_{1}^{2}}-2 r \frac{\sin \alpha \cos \alpha}{\sigma_{1} \sigma_{2}}+\frac{\sin ^{2} \alpha}{\sigma_{2}^{2}}, \quad r=\frac{a_{3}}{\sqrt{a_{1} a_{2}}}, \sigma_{1}^{2}=a_{1} b, \sigma_{2}^{2}=a_{2} b \text { and } \\
\alpha=\sqrt{k / m} t .
\end{gathered}
$$
density:

b) The distribution of the random variable $t^{-1} \varepsilon(t)$ converges to the distribution with the

$$
\begin{aligned}
& \rho(x)=\frac{m}{b \sqrt{a_{1} a_{2}-a_{3}^{2}}} \exp \left\{-\frac{x m\left(a_{1}+a_{2}\right)}{2 b\left(a_{1} a_{2}-a_{3}^{2}\right)}\right\} \otimes \\
& I_{0}\left(\frac{x m}{b\left(a_{1} a_{2}-a_{3}^{2}\right)} \sqrt{\frac{1}{4}\left(a_{1}-a_{2}\right)^{2}+a_{3}^{2}}\right), \quad x>0,
\end{aligned}
$$

where $I_{0}(x)$ is the modified Bessel function of the first kind with zero index and $\rho(x)=0$, when $x<0$.

Proof: We can write the solution of equation (2) in explicit form [2]:

$$
\begin{gathered}
u(t)=\frac{1}{\sqrt{k m}} \int_{0}^{t} f(s) g(w(s)) \sin (\sqrt{k / m}(t-s)) d w(s) \\
\dot{u}(t)=\frac{1}{m} \int_{0}^{t} f(s) g(w(s)) \cos (\sqrt{k / m}(t-s)) d w(s) .
\end{gathered}
$$

Let us introduce the parameter $T \geq T_{0}>0$ and denote

$$
u_{T}(t)=u(t T) / \sqrt{T}, \dot{u}_{T}(t)=\dot{u}(t T) / \sqrt{T} \text { and } w_{T}(t)=w(t T) / \sqrt{T} .
$$

Then,

$$
u_{T}(t)=\frac{1}{\sqrt{k m}}\left[\gamma_{T}^{(1)}(t) \sin (\sqrt{k / m} t T)-\gamma_{T}^{(2)}(t) \cos (\sqrt{k / m} t T)\right]
$$

and

$$
\dot{u}_{T}(t)=\frac{1}{m}\left[\gamma_{T}^{(1)}(t) \cos (\sqrt{k / m} t T)+\gamma_{T}^{(2)}(t) \sin (\sqrt{k / m} t T)\right]
$$

where

and

$$
\gamma_{T}^{(1)}(t)=\int_{0}^{t} g\left(w_{T}(s) \sqrt{T}\right) f(s T) \cos (\sqrt{k / m} s T) d w_{T}(s)
$$

$$
\gamma_{T}^{(2)}(t)=\int_{0}^{t} g\left(w_{T}(s) \sqrt{T}\right) f(s T) \sin (\sqrt{k / m} s T) d w_{T}(s) .
$$

Since each process $\gamma_{T}^{(i)}(t)$ for $i=1,2$ is a martingale with respect to the $\sigma$-algebra, $\sigma\left(w_{T}(s)\right.$, 
$s \leq t$ ), and since each satisfies the Skorohod condition of compactness of random processes [9], we can assume, without loss of generality, that $\gamma_{T}^{(i)}(t) \rightarrow \gamma^{(i)}(t)$ for $i=1,2$ and $w_{T}(t) \rightarrow w(t)$ in probability as $T \rightarrow \infty$ at every point $t>0$, where $w(t)$ is a Wiener process and each $\gamma^{(i)}(t)$ is a martingale with respect to the $\sigma$-algebra $\sigma(w(s), s \leq t)$.

Thus, (7) implies the convergencies

$$
u_{T}(t)-\frac{1}{\sqrt{k m}}\left[\gamma^{(1)}(t) \sin (\sqrt{k / m} t T)-\gamma^{(2)}(t) \cos (\sqrt{k / m} t T)\right] \rightarrow 0
$$

and

$$
\dot{u}_{T}(t)-\frac{1}{m}\left[\gamma^{(1)}(t) \cos (\sqrt{k / m} t T)+\gamma^{(2)}(t) \sin (\sqrt{k / m} t T)\right] \rightarrow 0
$$

in probability, as $T \rightarrow \infty$.

Consider now characteristics of martingales:

$$
\begin{gathered}
\left\langle\gamma_{T}^{(1)}(t)\right\rangle=\int_{0}^{t} g^{2}\left(w_{T}(s) \sqrt{T}\right) f^{2}(s T) \cos ^{2}(\sqrt{k / m} s T) d s \\
\left\langle\gamma_{T}^{(2)}(t)\right\rangle=\int_{0}^{t} g^{2}\left(w_{T}(s) \sqrt{T}\right) f^{2}(s T) \sin ^{2}(\sqrt{k / m} s T) d s \\
\left\langle\gamma_{T}^{(1)}(t), \gamma_{T}^{(2)}(t)\right\rangle=\frac{1}{2} \int_{0}^{t} g^{2}\left(w_{T}(s) \sqrt{T}\right) f^{2}(s T) \sin (2 \sqrt{k / m} s T) d s .
\end{gathered}
$$

Suppose that for the function $f^{2}(t)$ the first assumption of Theorem 1 is satisfied. It is easy to verify that, in this case,

$$
f^{2}(t) \cos ^{2}(\sqrt{k / m} t)=a_{0}+\alpha_{1}(t), f^{2}(t) \sin ^{2}(\sqrt{k / m} t)=a_{0}+\alpha_{2}(t)
$$

and

$$
\frac{1}{2} f^{2}(t) \sin (2 \sqrt{k / m} t)=\alpha_{3}(t)
$$

where $a_{0}=\frac{1}{4 L} \int_{0}^{2 L} f^{2}(s) d s$, and there is a constant $C>0$ that for all $t \geq 0$ satisfies the inequality,

$$
\left|\int_{0}^{t} \alpha_{i}(s)\right| \leq C, \quad i=1,2,3 .
$$

Then $\left\langle\gamma_{T}^{(i)}(t)\right\rangle=a_{0} \int_{0}^{t} g^{2}\left(w_{T}(s) \sqrt{T}\right) d s+\int_{0}^{t} g^{2}\left(w_{T}(s) \sqrt{t}\right) \alpha_{i}(s T) d s=I_{T}(t)+J_{T}(t)$.

Kulinich [6] implies $I_{T}(t) \rightarrow \beta(t)$ in probability as $T \rightarrow \infty$, where $\beta(t)=a_{0} b t$, and due to the Lemma, $E\left|J_{T}(t)\right| \rightarrow 0$. Therefore, $\left\langle\gamma_{T}^{(i)}(t)\right\rangle \rightarrow a_{0} b t$ in probability as $T \rightarrow \infty$ for $i=1,2$. And for the joint characteristic of martingales $\gamma_{T}^{(1)}$ and $\gamma_{T}^{(2)}(t)$, we have the equality,

$$
\left\langle\gamma_{T}^{(1)}(t), \gamma_{T}^{(2)}(t)\right\rangle=\int_{0}^{t} g^{2}\left(w_{T}(s) \sqrt{T}\right) \alpha_{3}(s T) d s,
$$

which, due to the Lemma, implies the convergence,

$$
E\left|\left\langle\gamma_{T}^{(1)}(t), \gamma_{T}^{(2)}(t)\right\rangle\right| \rightarrow 0 \text { as } t \rightarrow \infty
$$


Hence, for characteristics of the limit martingales we have

$$
\left\langle\gamma^{(i)}(t)\right\rangle=a_{0} b t, \quad i=1,2 \text { and }\left\langle\gamma^{(1)}(t), \gamma^{(2)}(t)\right\rangle=0 .
$$

It is easy to see that martingales $\gamma^{(1)}(t)$ and $\gamma^{(2)}(t)$ are continuous with probability 1 . Therefore, due to [3], there are independent Wiener processes $w^{(1)}(t)$ and $w^{(2)}(t)$ such that

$$
\gamma^{(1)}(t)=\sqrt{a_{0} b} w^{(1)}(t) \text { and } \gamma^{(2)}(t)=\sqrt{a_{0} b} w^{(2)}(t) .
$$

Thus, taking into consideration convergencies (8), we have

$$
\begin{gathered}
P\left\{u_{T}(1)<x_{1}, \dot{u}_{T}(1)<x_{2}\right\} \\
-P\left\{\sqrt{\frac{a_{0} b}{k m}} \zeta_{T}^{(1)}<x_{1}, \frac{\sqrt{a_{0} b}}{k m} \zeta_{T}^{(2)}<x_{2}\right\} \rightarrow 0 \text { as } T \rightarrow \infty,
\end{gathered}
$$

where

$$
\zeta_{T}^{(1)}=w^{(1)}(1) \sin (\sqrt{k / m} T)-w^{(2)}(1) \cos (\sqrt{k / m} T)
$$

and

$$
\left.\zeta_{T}^{(2)}=w^{(1)}(1) \cos (\sqrt{k / m} T)+w^{(2)}(1) \sin \sqrt{k / m} T\right) .
$$

Independence of the normally distributed random variables $w^{(1)}(1)$ and $w^{(2)}(1)$ implies that they have a bivariate normal distribution. Hence, due to [4], $\zeta_{T}^{(1)}$ and $\zeta_{T}^{(2)}$ are also bivariate normal for every $T$.

It is easy to verify, that for every $T$,

$$
E \zeta_{T}^{(i)}=0, \quad D \zeta_{T}^{(i)}=1 \text { and } E \zeta_{T}^{(1)} \zeta_{T}^{(2)}=0 .
$$

Therefore, the random variables, $\zeta_{T}^{(1)}$ and $\zeta_{T}^{(2)}$, are independent standard normal. Convergence (11) yields the proof of statement $1 a$ ) of Theorem 1.

Since for instantaneous energy $\varepsilon(t)$ in system (2) we have the equality,

$$
T^{-1} \varepsilon(T)=\frac{1}{2 m}\left(\left[\gamma_{T}^{(1)}(1)\right]^{2}+\left[\gamma_{T}^{(2)}(1)\right]^{2}\right)
$$

then, for all $x>0$,

$$
\lim _{T \rightarrow \infty} P\left\{T^{-1} \varepsilon(T)<x\right\}=P\left\{\frac{a_{0} b}{2 m}\left(\left[w^{(1)}(1)\right]^{2}+\left[w^{(2)}(1)\right]^{2}\right)<x\right\} .
$$

According to Gnedenko [4], the random variable $\left[w^{(1)}(1)\right]^{2}+\left[w^{(2)}(1)\right]^{2}$ has a $\chi^{2}$ distribution with two degrees of freedom and it coincides with the exponential distribution with parameter $1 / 2$. Hence, the distribution of the random variable $T^{-1} \varepsilon(T)$, as $T \rightarrow \infty$ converges to the exponential distribution with parameter $m\left[a_{0} b\right]^{-1}$. This proves statement $1 b$ ) of Theorem 1 .

Next, suppose that $2 L=n_{0} \pi \sqrt{m / k}$ and that at least one of the constants, $c_{0}$ or $a_{3}$, is not equal to zero. Then (9) can be represented in the form:

$$
f^{2}(t) \cos ^{2}(\sqrt{k / m} t)=a_{1}+\alpha_{1}(t), f^{2}(t) \sin ^{2}(\sqrt{k / m} t)=a_{2}+\alpha_{2}(t)
$$

and

$$
\frac{1}{2} f^{2}(t) \sin (2 \sqrt{k / m} t)=a_{3}+\alpha_{3}(t) .
$$


Therefore, in this case we have

and

$$
\left\langle\gamma_{T}^{(i)}(t)\right\rangle=a_{i} \int_{0}^{t} g^{2}\left(w_{T}(s) \sqrt{T}\right) d s+\int_{0}^{t} g^{2}\left(w_{T}(s) \sqrt{T}\right) \alpha_{i}(s T) d s, \quad i=1,2
$$

$$
\left\langle\gamma_{T}^{(1)}(t), \gamma_{T}^{(2)}(t)\right\rangle=a_{3} \int_{0}^{t} g^{2}\left(w_{T}(s) \sqrt{T}\right) d s+\int_{0}^{t} g^{2}\left(w_{T}(s) \sqrt{T}\right) \alpha_{3}(s T) d s .
$$

As in the proof of statement 1 of Theorem 1, we obtain characteristics of the limit martingales:

$$
\left\langle\gamma^{(1)}(t)\right\rangle=a_{1} b t,\left\langle\gamma^{(2)}(t)\right\rangle=a_{2} b t \text { and }\left\langle\gamma^{(1)}(t), \gamma^{(2)}(t)\right\rangle=a_{3} b t
$$

Also,

$$
\gamma^{(i)}(t)=\sqrt{b}\left[b_{i 1} w^{(1)}(t)+b_{i 2} w^{(2)}(t)\right], \quad i=1,2,
$$

where $w^{(1)}(t)$ and $w^{(2)}(t)$ are independent Wiener processes and $\left(b_{i 1}, b_{i 2}\right)$ is the $i$-th row of the matrix $B^{1 / 2}$, where $B=\left(\begin{array}{ll}a_{1} & a_{3} \\ a_{3} & a_{2}\end{array}\right)$. The independence of the Wiener processes, $w^{(1)}(t)$ and $w^{(2)}(t)$, implies that random variables $\gamma^{(1)}(1)$ and $\gamma^{(2)}(1)$ have normal distributions with parameters $\left(0, \sigma_{i}^{2}\right)$, where $\sigma_{1}^{2}=a_{1} b$ and $\sigma_{2}^{2}=a_{2} b$ are bivariate normal with the coefficient of correlation $r=a_{3}\left(a_{1} a_{2}\right)^{-1}$. Hence, according to Gnedenko [4], the joint density of the random variables,

$$
\gamma^{(1)}(1) \sin (\sqrt{k / m} T)-\gamma^{(2)}(1) \cos (\sqrt{k / m} T)
$$

and

$$
\left.\gamma^{(1)}(1) \cos (\sqrt{k / m} T)+\gamma^{(2)}(1) \sin \sqrt{k / m} T\right)
$$

is of the form (5) with $t=T$. To complete the proof of statement $2 a$ ) of Theorem 1 , we use convergencies (8). Equality (12) implies that the limit distribution of the random variable $T^{-1} \varepsilon(T)$ coincides with the distribution of the absolute value of a bivariate normal random vector.

Corollary: Under the conditions of Theorem 1 ,

$$
\begin{gathered}
\lim _{t \rightarrow \infty}\left(E t^{-1} \varepsilon(t)\right)=\frac{b}{2 m}\left(a_{1}+a_{2}\right) ; \\
\lim _{t \rightarrow \infty} D t^{-1} \varepsilon(t)=\frac{b^{2}}{2 m^{2}}\left(a_{1}^{2}+a_{2}^{2}\right), \text { while } a_{3}=0 ; \\
\lim _{t \rightarrow \infty} D t^{-1} \varepsilon(t)=\frac{b^{2}}{2 m^{2}}\left(a_{0}^{2}+\frac{3}{2} a_{3}^{2}\right), \text { while } a_{3} \neq 0 \text { and } c_{0}=0 ; \\
\lim _{t \rightarrow \infty} D t^{-1} \varepsilon(t)=\frac{b^{2}}{2 m^{2}}\left(a_{1}^{2}+a_{2}^{2}+3 a_{3}^{2}+\beta\right), \text { while } a_{3} \neq 0 \quad c_{0} \neq 0 \text { and } \\
\beta=\frac{2 a_{3}^{2}}{\left(a_{1}-a_{2}\right)^{4}}\left\{4 a_{3}^{2}\left[a_{1}^{4}-a_{1}^{2} \sqrt{a_{1}^{4}-\left(a_{1}-a_{2}\right)^{2}}-\frac{1}{2}\left(a_{1}-a_{2}\right)^{2}\right]\right. \\
\left.-\left[a_{1}^{4}-\left(a_{1}^{2}-a_{2}^{2}\right) \sqrt{a_{1}^{4}-\left(a_{1}-a_{2}\right)^{2}}\right]\right\}+2\left(a_{1} a_{2} a_{3}\right)^{2}+a_{3}^{2} .
\end{gathered}
$$

In this case we can change the order of limit and expectation (variance). We use the latter, the explicit form of the limit value $\gamma^{(i)}(1)$ for every $i$ and equality (12) to prove the statement. 
Theorem 2: Let the function $g(x)$ in equation (2) have a second derivative almost everywhere and for some $\alpha>0$ satisfy the conditions:

and

$$
\lim _{|x| \rightarrow \infty}\left(\frac{1}{|x|^{\alpha}} \int_{0}^{x} g^{2}(v) d v-b(x)\right)=0, \text { with } b(x)= \begin{cases}b_{1}, & x>0 \\ b_{2}, & x<0\end{cases}
$$

Then

$$
\lim _{|x| \rightarrow \infty} \frac{1}{|x|^{\alpha}} \int_{0}^{x}\left|g^{\prime}(v)+g(v) g^{\prime \prime}(v)\right| d v=0
$$

$$
P\left\{\frac{u(t)}{t^{(\alpha+1) / 4}}<x_{1}, \frac{\dot{u}(t)}{t^{(\alpha+1) / 4}}<x_{2}\right\}-P\left\{v(t)<x_{1}, \dot{v}(t)<x_{2}\right\} \rightarrow 0 \text { as } t \rightarrow \infty,
$$

where $v(t)$ is the position and $\dot{v}(t)$ is the velocity of the homogeneous harmonic oscillator

$$
m \ddot{v}(t)+k v(t)=0, \quad t>0
$$

with the initial condition

$$
v(0)=-\frac{1}{\sqrt{k m}} \gamma^{(2)}(1) \text { and } \dot{v}(0)=\frac{1}{m} \gamma^{(1)}(1) .
$$

Here each $\gamma^{(i)}(t)$ is a martingale with respect to the $\sigma$-algebra $\sigma(w(s), s \leq t)$ with characteristics:

$$
\begin{gathered}
\left\langle\gamma^{(i)}(t)\right\rangle=a_{i} \beta(t), \quad i=1,2 \text { and }\left\langle\gamma^{(1)}(t), \gamma^{(2)}(t)\right\rangle=a_{3} \beta(t), \\
\text { while } \beta(t)=\alpha \int_{0}^{t}|w(s)|^{\alpha-1} b(w(s)) \operatorname{sign} w(s) d s .
\end{gathered}
$$

Proof: The proof is similar to that of $(8)$ in Theorem 1, with the difference that, in this case,

and

$$
\begin{gathered}
u_{T}(t)=T^{-(\alpha+1) / 4} u(t T), \quad \dot{u}_{T}(t)=T^{-(\alpha+1) / 4} \dot{u}(t T), \\
\gamma_{T}^{(1)}(t)=T^{(1-\alpha) / 4} \int_{0}^{t} g\left(w_{T}(s) \sqrt{T}\right) f(s T) \cos (\sqrt{k / m} s T) d w_{T}(s),
\end{gathered}
$$

with characteristics

$$
\gamma_{T}^{(2)}(t)=T^{(1-\alpha) / 4} \int_{0}^{t} g\left(w_{T}(s) \sqrt{T}\right) f(s T) \sin (\sqrt{k / m} s T) d w_{T}(s)
$$

and

$$
\left\langle\gamma_{T}^{(i)}(t)\right\rangle=a_{i} T^{(1-\alpha) / 2} \int_{0}^{t} g^{2}\left(w_{T}(s) \sqrt{T}\right) d s+T^{(1-\alpha) / 2} \int_{0}^{t} g^{2}\left(w_{T}(s) \sqrt{T}\right) \alpha_{i}(s T) d s, \quad i=1,2,
$$

$$
\left\langle\gamma_{T}^{(1)}(t), \gamma_{T}^{(2)}(t)\right\rangle=a_{3} T^{(1-\alpha) / 2} \int_{0}^{t} g^{2}\left(w_{T}(s) \sqrt{T}\right) d s+T^{(1-\alpha) / 2} \int_{0}^{t} g^{2}\left(w_{T}(s) \sqrt{T}\right) \alpha_{3}(s T) d s .
$$

Due to the Lemma, 
and

$$
\left\langle\gamma_{T}^{(i)}(t)\right\rangle=a_{i} T^{(1-\alpha) / 2} \int_{0}^{t} g^{2}\left(w_{T}(s) \sqrt{T}\right) d s+o(1)
$$

$$
\left\langle\gamma_{T}^{(1)}(t), \gamma_{T}^{(2)}(t)\right\rangle=a_{3} T^{(1-\alpha) / 2} \int_{0}^{t} g^{2}\left(w_{T}(s) \sqrt{T}\right) d s+o(1)
$$

where $o(1)$ is such that $E|o(1)| \rightarrow 0$ as $T \rightarrow \infty$ for all $t>0$. Next, Kulinich [6] established that

in probability as $T \rightarrow \infty$, where

$$
T^{(1-\alpha) / 2} \int_{0}^{t} g^{2}\left(w_{T}(s) \sqrt{T}\right) d s \rightarrow \beta(t)
$$

$$
\beta(t)=2\left[\int_{0}^{w(t)}|v|^{\alpha} b(v) d v-\int_{0}^{t}|w(s)|^{\alpha} b(w(s)) d w(s)\right] .
$$

Since $\alpha>0$, using Itô's formula, we have

Hence,

$$
\beta(t)=\alpha \int_{0}^{t}|w(s)|^{\alpha-1} b(w(s)) \operatorname{sign} w(s) d s .
$$

$$
\left\langle\gamma_{T}^{(i)}(t)\right\rangle \rightarrow a_{i} \beta(t), \quad i=1,2 \text { and }\left\langle\gamma_{T}^{(1)}(t), \gamma_{T}^{(2)}(t)\right\rangle \rightarrow a_{3} \beta(t)
$$

in probability as $T \rightarrow \infty$. Thus, we obtain convergence (8), where each $\gamma^{(i)}(t)$ is a continuous, with probability 1 , martingale with respect to $\sigma(w(s), s \leq t)$, with characteristics:

$$
\left\langle\gamma^{(i)}(t)\right\rangle=a_{i} \beta(t), i=1,2 \text { and }\left\langle\gamma^{(1)}(t)(t), \gamma^{(2)}(t)\right\rangle=a_{3} \beta(t)
$$

where $\beta(t)$ has the form (14). Using convergence (8) for $t=1$ and an explicit form of the solution of problem (13), we complete the proof of Theorem 2 .

Corollary: Under the conditions of Theorem 2,

$$
\lim _{t \rightarrow \infty} E t^{-(\alpha+1) / 2} \varepsilon(t)=\frac{\alpha\left(a_{1}+a_{2}\right)}{2 m} \int_{0}^{1} E|w(s)|^{\alpha-1} b(w(s)) \operatorname{sign} w(s) d s .
$$

This equality is a consequence of the following statements:

1) the equality (12);

2) the equality $E\left[\gamma_{T}^{(i)}(t)\right]^{2}=E\left\langle\gamma_{T}^{(i)}(t)\right\rangle$

3) the possibility to change the order of limit and expectation.

Remark: Let $q\left(x_{1}, x_{2}\right)$ be a joint density of the distribution of $\gamma^{(1)}(1)$ and $\gamma^{(2)}(1)$ and $\rho_{t}\left(x_{1}, x_{2}\right)$ be a joint density of the distribution of the position $v(t)$ and the velocity $\dot{v}(t)$ at the moment $t$, described by (13). Then,

$$
\begin{gathered}
\rho_{t}\left(x_{1}, x_{3}\right)=q\left[x_{1} \sqrt{k m} \sin (\sqrt{k / m} t)+x_{2} m \cos (\sqrt{k / m} t)\right. \\
\left.-x_{1} \sqrt{k m} \cos (\sqrt{k / m} t)+x_{2} m \sin (\sqrt{k / m} t)\right] m \sqrt{k m}
\end{gathered}
$$

Using the explicit form of the solution to equation (13) we get 


$$
\gamma^{(1)}(1)=v(t) \sqrt{k m} \sin (\sqrt{k / m} t)+\dot{v}(t) m \cos (\sqrt{k / m} t)
$$

and

$$
\gamma^{(2)}(1)=-v(t) \sqrt{k m} \cos (\sqrt{k / m} t)+\dot{v}(t) m \sin (\sqrt{k / m} t)
$$

which yields (15).

\section{References}

[1] Bendersky, M.M. and Pastur, L.A., On asymptotics of solutions of the second order equation with random coefficients, Theor. Func., Functional. Analiz i Primeneniya 22 (1975), 3-14.

[2] Dyvnich, N.T., On limit behavior of solutions of the Cauchy problem for equation of heat conductivity disturbed by white noise process, Ukrain. Math. J. 29:5 (1977), 646-650.

[3] Gihman, I.I. and Skorokhod, A.V., The Theory of Stochastic Processes, III, Nauka, Moscow 1975. English transl. Springer-Verlag, Berlin 1978.

[4] Gnedenko, B.V., Probability Theory, Nauka, Moscow 1977.

[5] Krylov, N.V., Controlled Diffusion Process, Nauka, Moscow 1977.

[6] Kulinich, G.L., On asymptotic behavior of distributions $\int_{0}^{t} g(w(s)) d s$-type functionals for diffusion processes, Theor. Veroyatnost. i Mat. Statist. 8 (1975), 95-101.

[7] Kulinich, G.L., On limit behavior of random harmonic oscillator, Vestnik Kiev. Univ. (Math. \& Mech.) 25 (1983), 108-112.

[8] Papanicolau, G.C., Stochastic Equations and Their Applications, American Math., Mont. 80:5 (1973), 526-545.

[9] Skorokhod, A.V., The Study on Theory of Random Processes, Izdatelstvo Kiev. Univ. 1961. English Transl. Addison-Wesley, Reading, MA 1965. 


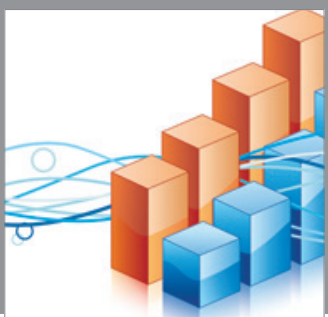

Advances in

Operations Research

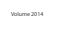

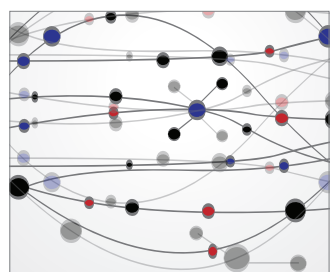

\section{The Scientific} World Journal
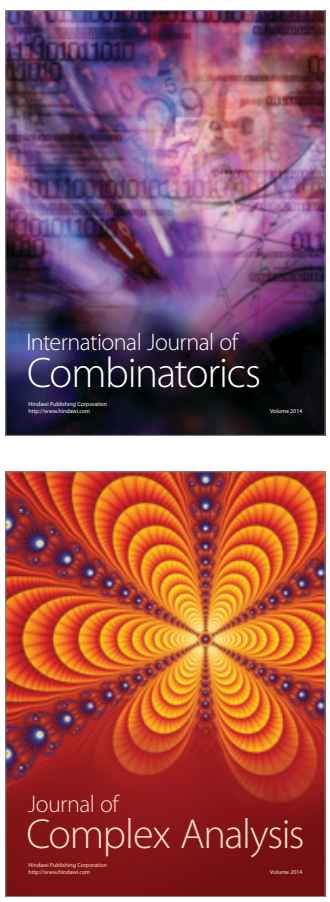

International Journal of

Mathematics and

Mathematical

Sciences
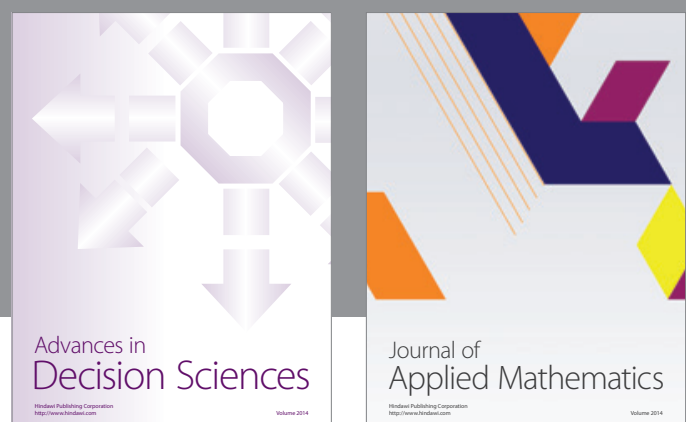

Journal of

Applied Mathematics
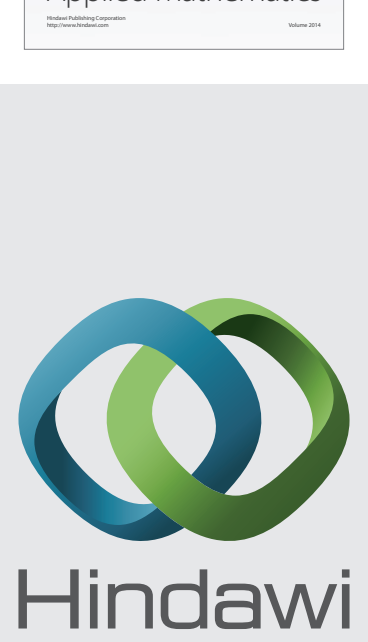

Submit your manuscripts at http://www.hindawi.com
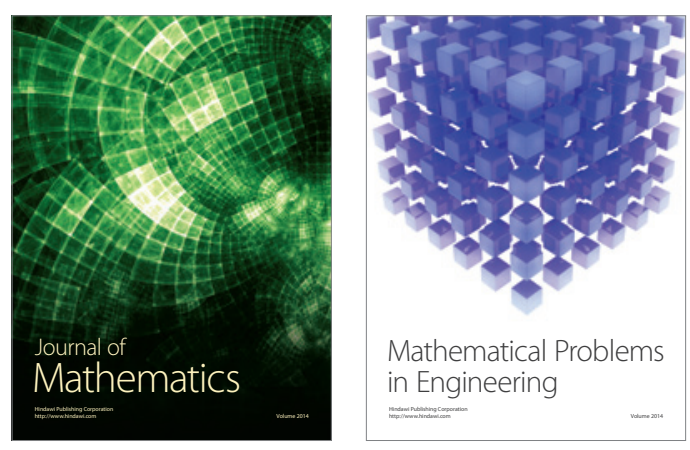

Mathematical Problems in Engineering
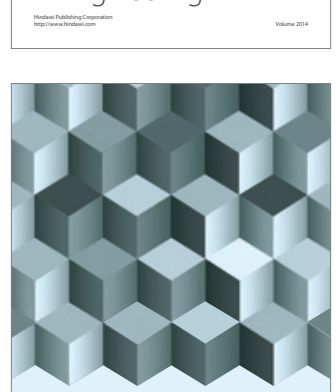

Journal of

Function Spaces
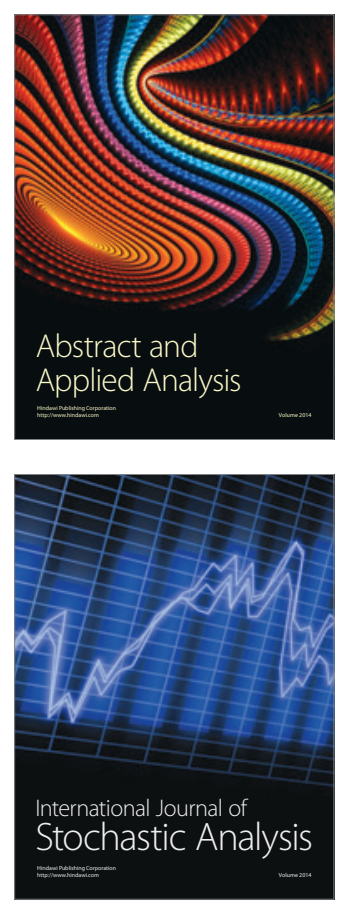

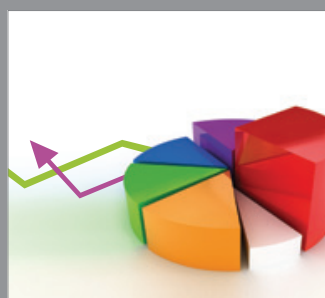

ournal of

Probability and Statistics

Promensencen
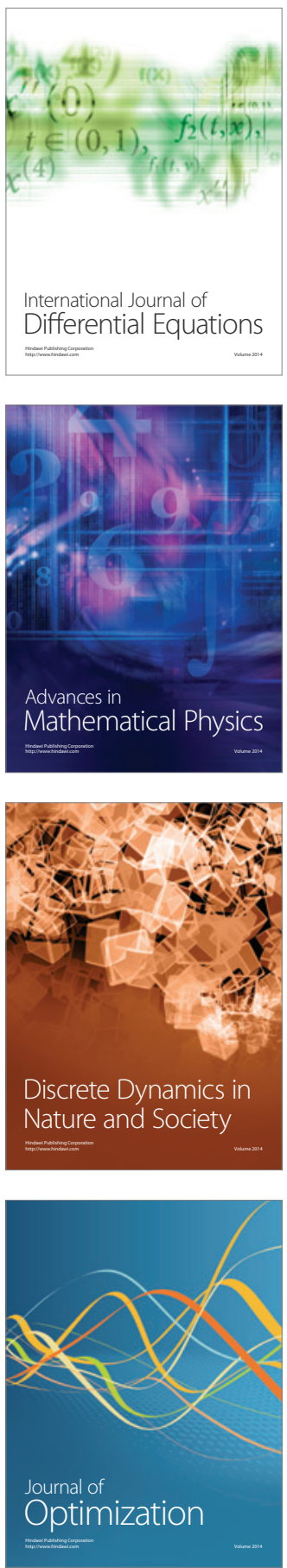